\title{
Endoaortic Clamping Does Not Increase the Risk of Stroke in Minimal Access Mitral Valve Surgery: A Multicenter Experience
}

\author{
Filip Casselman, MD, PhD, Jose Aramendi, MD, Mohamed Bentala, MD, \\ Pascal Candolfi, PhD, Rudolf Coppoolse, MD, Borut Gersak, MD, PhD, \\ Ernesto Greco, MD, PhD, Paul Herijgers, MD, PhD, Steven Hunter, MD, \\ Ralf Krakor, MD, PhD, Mauro Rinaldi, MD, Frank Van Praet, MD, \\ Geert Van Vaerenbergh, ECP, and Joseph Zacharias, MD
}

\begin{abstract}
Department of Cardiovascular \& Thoracic Surgery, OLV, Aalst, Belgium; Department of Cardiac Surgery, Hospital de Cruces, Bilbao, Spain; Department of Cardiothoracic Surgery, Amphia Breda Hospital, Breda, The Netherlands; Biostatistics, Edwards Lifesciences, Nyon, Switzerland; Department of Cardiac Surgery, Schûchtermann Klinik, Bad Rothenfelde, Germany; Department of Cardiovascular Surgery, University Medical Centre, Ljubljana, Slovenia; Department of Cardiac Surgery, Policlinico Umberto I, Rome, Italy; Department of Cardiac Surgery, Gasthuisberg, Leuven, Belgium; Department of Cardiac Surgery, Northern General Hospital, Sheffield, United Kingdom; Department of Cardiac Surgery, Klinikum Dortmund, Dortmund, Germany; Department of Cardiac Surgery, Azienda Ospedaliera San Giovanni Battista, Turin, Italy; and Department of Cardiac Surgery, Victoria Hospital, Blackpool, United Kingdom
\end{abstract}

Background. Some controversy exists regarding the safety of endoaortic balloon clamping in minimal access isolated mitral valve surgery (MIMVS). The aim of this European multicenter study was to analyze the results in 10 experienced centers and compare the outcomes with published data.

Methods. The most recent 50 consecutive MIMVS cases from10 European surgeons who had performed at least $\mathbf{1 0 0}$ procedures were prospectively collected and retrospectively analyzed. All procedures were performed through right minithoracotomy with femoral cannulation and endoaortic balloon occlusion. In-hospital and 30-day outcomes were studied. Mortality and stroke rates were compared with published median sternotomy and MIMVS outcomes.

Results. Mean age was $63.2 \pm 12.5$ years, $289(57.8 \%)$ were male, mean logistic European system for cardiac operative risk evaluation was $6.1 \pm 6.2$, and $53(10.6 \%)$ procedures had cardiac reoperations. Concomitant procedures were performed in $126(25.9 \%)$ cases. Three patients $(0.6 \%)$ required conversion to full sternotomy. Ten patients $(2.0 \%)$ necessitated endoaortic balloon clamping conversion (8 to external clamping), and

$\mathrm{D}$ espite excellent operative results in mitral valve surgery using standard sternotomy [1], a gradual interest has emerged to limit the invasiveness of the procedure. As such, minimally invasive mitral valve surgery (MIMVS) was developed in the late 1990s [2].

Since then, several methods have been used to clamp the ascending aorta. Of these, the endoaortic balloon and

Accepted for publication April 1, 2015.

Address correspondence to Dr Casselman, Department of Cardiovascular and Thoracic Surgery, OLV Clinic, Moorselbaan 164, B-9300 AALST, Belgium; e-mail: filip.casselman@olvz-aalst.be. re-exploration for bleeding was necessary in $24 \mathbf{( 4 . 8 \% )}$ cases. Mean aortic cross-clamp and cardiopulmonary bypass times were $85.6 \pm 30.1$ and $129.5 \pm 40.2$ min, respectively, and were significantly longer for concomitant procedures $(p<0.001)$. There were no aortic dissections and no deep venous thromboses. Operative mortality (none neurologic) and major stroke occurred in $7(1.4 \%)$ and $4(0.8 \%)$ patients, respectively. These rates compared favorably with the published literature on isolated primary mitral valve surgery (MVS) through sternotomy or minithoracotomy (mortality rates $0.2 \%$ to $11.6 \%$, stroke rates $0.6 \%$ to $4.4 \%$ ).

Conclusions. Once procedural proficiency is acquired, endoaortic balloon clamping in MIMVS is a safe and effective technique. Despite the fact that this patient cohort also includes combined and redo procedures, the observed mortality and stroke rate compared favorably with the existing literature on primary isolated mitral valve surgery irrespective of the approach.

(Ann Thorac Surg 2015;100:1334-9) (C) 2015 by The Society of Thoracic Surgeons

external aortic clamp are the most frequently used techniques.

Recently, some concerns were raised about inferior outcomes related to the use of the endoaortic balloon in MIMVS [3]. This information was not confirmed by prospective studies but a concern about MIMVS was also raised in a consensus paper by the International Society for Minimally Invasive Cardiac Surgery (ISMICS ) where an increased stroke rate was noted [4].

The aim of the current retrospective study was to analyze the perioperative safety and outcomes associated with the use of an endoaortic balloon. In addition, we investigated 
the stroke rate associated with MIMVS across Europe, and compared these outcomes with the existing literature.

\section{Material and Methods}

\section{Study Population}

Ten centers across Europe which perform MIMVS using the endoaortic balloon (Edwards Lifesciences, Irvine, CA) and which were beyond the learning curve (defined as an experience $>50$ cases) but had performed greater than 100 cases in total (Table 1) were asked to contribute their last 50 consecutive cases (excluding their first 50 cases) to a database to produce a total study population of $n=500$. The main reasons for preferring the endoaortic balloon technique were absence of an extra port and direct endoaortic cardioplegia administration, avoiding an extra purse-string suture on the ascending aorta. All data were extracted retrospectively from the various institutional databases. The MIMVS was defined as any mitral repair or replacement; concomitant atrial fibrillation surgery and or tricuspid valve surgery were also included. Reoperative cardiac procedures were not excluded. Patients who had an inadequate iliac access for endoballoon insertion or retrograde arterial perfusion were excluded. Other reasons for exclusion were the following: aortic regurgitation greater than grade 2; an ascending aortic diameter greater than $40 \mathrm{~mm}$; previous right lobectomy; extensive pleural adhesions (discovered perioperatively); and previous aorto-bifemoral or bi-iliac grafting. Usually emergency procedures are excluded although some centers do not take this as an exclusion criterion. Each individual center obtained ethical committee approval for this study but due to the retrospective and anonymous nature of the study, individual patient consent was waived in all centers.

\section{Operative Technique}

All patients were operated through a right minithoracotomy with peripheral venous and arterial cannulation,

Table 1. Centers' Experience With Minimally Invasive Mitral Valve Surgery Prior to Case Inclusion in the Present Study

\begin{tabular}{lcc}
\hline Center & $\begin{array}{c}\text { Institutional Endoballoon } \\
\text { Experience Prior to the Study }\end{array}$ & $\begin{array}{c}\text { Institutional \% } \\
\text { of MIMVS }\end{array}$ \\
\hline 1 & 2338 & 91 \\
2 & 51 & 70 \\
3 & 450 & 99 \\
4 & 450 & 99 \\
5 & 870 & 12 \\
6 & 360 & 80 \\
7 & 136 & 85 \\
8 & 345 & 85 \\
9 & 1000 & 99 \\
10 & 529 & 94 \\
\hline
\end{tabular}

MIMVS = minimally invasive mitral valve surgery (vs total cases of isolated mitral valve surgery in the department). and endoaortic balloon occlusion (EndoClamp or IntraClude; Edwards Lifesciences, Irvine, CA). Five centers systematically use double (jugular and femoral) venous cannulation, whereas the others use single venous femoral cannulation in isolated mitral valve surgery and double venous cannulation if tricuspid valve surgery is associated. The operative approach for MIMVS performed through a minithoracotomy has been previously described [5]. All centers use intraoperative transesophageal echocardiography monitoring. Figure 1 demonstrates the operative setup. Cardiac arrest was obtained using intermittent cold antegrade crystalloid or blood cardioplegia ( $50.0 \%$ vs $49.8 \%$, respectively). Retrograde cardioplegia was not used. The nature of the cardioplegic solution or time intervals of reinfusion was administered according to individual institutional protocols. Both caval veins were occluded if the right atrium was opened.

Six centers performed atrial fibrillation ablation surgery; 4 using cryoablation and 2 using monopolar radiofrequency energy. The lesion set was a full left-sided Cox-Maze lesion set; box lesion with extension to the base of the left atrial appendage and a left isthmus lesion. Two centers closed the left atrial appendage internally in case of ablation.

\section{Data Collection}

Using similar templates, demographic data and preoperative assessment were recorded and reviewed for any significant discrepancy. Major clinical outcome measures included cardiopulmonary bypass time (CPB), crossclamp time, type of surgery, and perioperative events such as full sternotomy conversion. Mortality and major complications (in-hospital and up to 30 days) such as stroke (modified Rankin Scale) [6], reoperation for bleeding, respiratory failure, or severe infection were collected. Echocardiographic data were recorded before, and up to, 30 days after surgery. Definitions of these complications were clear and accepted by all centers, primarily based on The Society of Thoracic Surgeons

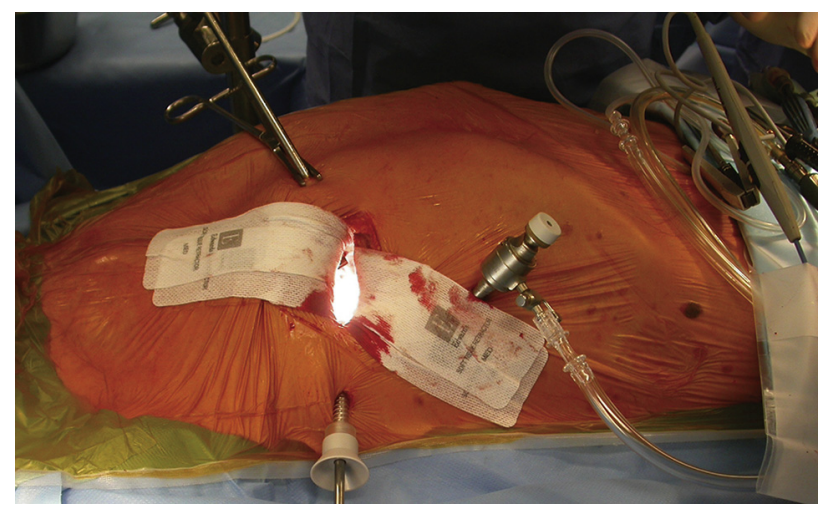

Fig 1. View of the operative setup for minimally invasive mitral valve surgery. Note the working port, the left atrial retractor shaft (top), venting position (right from the working port), and endoscope (under the working port). 
score definition. The definition of all outcome variables is available in the Appendix.

\section{Statistical Analysis}

Quantitative continuous variables are described with means \pm standard deviation and quantitative discrete variables with absolutes and relatives frequencies. Inference statistics comparing continuous variables are made using the Student $t$ test and the Wilcoxon rank sum test as appropriate. To compare discrete variables, the Pearson $\chi^{2}$ test was applied. Two-sided tests were utilized and a type I error significance level of 0.05 was considered. Early events ( $\leq 30$ days post-implantation) were calculated as simple percentages (number of complications divided by number of patients). All analyses were performed with the use of $R$ software, version 2.13.1 [7].

\section{Results}

All participating centers had experience with MIMVS using the endoballoon technique prior to starting this study. The respective centers' experience is summarized in Table 1.

\section{Study Population}

A total of 500 patients equally distributed among the participating centers were included. Baseline patient demographic characteristics are summarized in Table 2. Previous cardiac surgery was reported in 53 patients $(10.6 \%)$ and consisted of mitral valve surgery in 33 patients $(6.6 \%)$, coronary artery bypass grafting (CABG) in 18 cases $(3.6 \%)$, and aortic valve surgery in 13 cases $(2.6 \%)$. A combined procedure was performed in 126 patients.

\section{Surgical Procedure}

Perioperative details are presented in Tables 3 and 4. Concomitant procedures (atrial fibrillation surgery or tricuspid valve) were performed in 126 patients (140 procedures) $(25.4 \%)$. The IntraClude device was used in $70.2 \%$ of cases. The CPB and cross-clamp times were prolonged in this group versus patients without concomitant procedures; CPB time $153.8 \pm 43.7$ versus $120.8 \pm 34.8 \mathrm{~min}(p<0.001)$, and cross-clamp time $98.1 \pm$

Table 2. Baseline Characteristics

\begin{tabular}{lc}
\hline Characteristic & $\begin{array}{c}\text { MIMVS } \\
\text { (500 Patients) }\end{array}$ \\
\hline Age (years, mean $\pm \mathrm{SD})$ & $63.2 \pm 12.5$ \\
Male sex $(\%)$ & $289(57.8 \%)$ \\
Body mass index $\left(\mathrm{kg} / \mathrm{m}^{2}\right.$, mean $\left.\pm \mathrm{SD}\right)$ & $25.9 \pm 4.3$ \\
Preoperative atrial fibrillation & $32.4 \%$ \\
Logistic EuroSCORE 1 (mean $\pm \mathrm{SD})$ & $6.1 \% \pm 6.2 \%$ \\
Previous cardiac operation & $10.6 \%$ \\
NYHA $\geq$ III or IV & $263 / 499(52.7 \%)$ \\
\hline
\end{tabular}

EuroSCORE $=$ European system for cardiac operative risk evaluation; MIMVS = minimally invasive mitral valve surgery; $\quad$ NYHA $=$ New York Heart Association functional class.
Table 3. Surgical Procedure

\begin{tabular}{lc}
\hline Surgical Procedure & $\begin{array}{c}\text { MIMVS } \\
\text { (500 Patients) }\end{array}$ \\
\hline Replacement & $131(26.2 \%)$ \\
Bioprosthesis & $32 / 131(24.4 \%)$ \\
Mechanical & $99 / 131(75.6 \%)$ \\
Repair & $369(73.8 \%)$ \\
Commissurotomy & $10 / 369(2.7 \%)$ \\
Ring annuloplasty & $363 / 369(98.4 \%)$ \\
Chordae surgery & $20 / 369(5.4 \%)$ \\
Concomitant procedures & \\
Tricuspid valve surgery & $70 / 500(14.0 \%)$ \\
Atrial fibrillation ablation & $70 / 500(14.0 \%)$ \\
Antegrade cardioplegia & \\
Blood & $249 / 500(49.8 \%)$ \\
Crystalloid & $250 / 500(50.0 \%)$ \\
\hline
\end{tabular}

MIMVS $=$ minimally invasive mitral valve surgery.

32.7 versus $81.1 \pm 27.6 \min (p<0.001)$. Clamping conversion was observed in $2.0 \%$ (10 patients; 8 patients required a direct aortic clamping and 2 patients were further operated on fibrillating heart) (Table 4). Surgical conversion to full sternotomy was reported in 3 patients $(0.6 \%)$ : 1 patient underwent a mitral valve repair with unsatisfactory result and underwent a conversion to sternotomy for valve replacement; another patient underwent also an unsatisfactory mitral valve repair followed by valve replacement during the same clamping time but with ischemia during CPB weaning. This patient underwent a conversion to sternotomy for additional CABG. This patient ultimately died of severe multiple organ failure postoperatively. The reason for the third conversion was unclear from the retrospective analysis.

\section{Clinical Outcomes}

Thirty-day or in-hospital outcomes are summarized in Table 5 . The observed mortality rate was $1.4 \%(n=7)$. No aortic dissection occurred and the incidence of postoperative hemorrhage requiring re-exploration was $4.8 \%$ ( $\mathrm{n}=24$ patients). Major stroke (defined as modified

Table 4. Intraoperative Variables

\begin{tabular}{lc}
\hline Variable & MIMVS \\
(500 Patients)
\end{tabular}

$\mathrm{CPB}=$ cardiopulmonary bypass; MIMVS $=$ minimally invasive mitral valve surgery. 
Table 5. Major Postoperative Outcome

\begin{tabular}{lc}
\hline 30-Day Outcomes & $\begin{array}{c}\text { MIMVS } \\
\text { (500 Patients) }\end{array}$ \\
\hline Mortality & $7 / 500(1.4 \%)$ \\
Morbidity & \\
$\quad$ Aortic dissection & 0 \\
Reoperation for bleeding & $24 / 500(4.8 \%)$ \\
Transfusion postoperative & $159 / 500(31.8 \%)$ \\
Deep venous thrombosis & 0 \\
TIA & $3 / 500(0.6 \%)$ \\
Permanent stroke & $4 / 500(0.8 \%)$ \\
Renal failure & $13 / 500(2.6 \%)$ \\
Groin lymphocele & $7 / 500(1.4 \%)$ \\
Myocardial infarction & $5 / 500(1 \%)$ \\
\hline MIMVS = minimally invasive mitral valve surgery; & TIA = transitory
\end{tabular}
ischemic attack.

Rankin Score MRS $\geq 2$ ) was observed in 4 patients $(0.8 \%)$. Transitory ischemic attack occurred in 3 patients $(0.6 \%)$ resulting in a total neurologic event rate of $1.4 \%$. Renal failure was observed in $2.6 \%$. The incidence of a new myocardial infarction was $1.0 \%$ (5 patients).

\section{Echocardiographic Data}

Table 6 summarizes the 30-day echocardiographic findings. The cardiac function was preserved and mitral and tricuspid valve function restored.

\section{Literature Comparison}

We compared the results to the currently available literature on isolated mitral valve surgery either minimally invasively or through full sternotomy [1, 2, 4, 8-19]. Mortality rates reported were anywhere between 0.2 for elective mitral valve repair [19] to $11.6 \%$ for valve replacement [9] versus an observed mortality rate of $1.4 \%$ in the current analysis including valve replacement, combined and reoperative cases. The reported major stroke rates in elective mitral valve repair were between $0.6 \%$ [19] and $4.4 \%$ [11] versus an observed major stroke rate of $0.8 \%$ in our study population including valve replacement, combined and reoperative procedures.

\section{Comment}

Despite the fact that MIMVS has now been around for 20 years $[2,20]$, its penetration or adoption rate remains

Table 6. Echocardiographic Data

\begin{tabular}{lcc}
\hline Echocardiographic Findings & Baseline & 30 Days \\
\hline $\begin{array}{l}\text { MR (moderate to severe }-3 \\
\quad \text { or severe }-4)\end{array}$ & $445 / 500(89.0 \%)$ & $3 / 396(0.8 \%)$ \\
$\begin{array}{l}\text { TR (moderate to severe }-3 \\
\quad \text { or severe }-4)\end{array}$ & $57 / 499(11.4 \%)$ & $8 / 396(2.0 \%)$ \\
Ejection fraction & $0.569 \pm 0.112$ & $0.541 \pm 0.094$ \\
\hline
\end{tabular}

$\mathrm{MR}=$ mitral valve regurgitation;

$\mathrm{TR}=$ tricuspid valve regurgitation. relatively low. Indeed, a report from The STS database indicated that MIMVS was performed in only $20.1 \%$ of isolated mitral valve surgery in 2008 [21]. Various reasons may account for this. First, the transition to MIMVS requires additional training, even for established practices of mitral valve surgery. In addition, the need for different perfusion cannulas, video-assistance, and surgical instruments require an investment from teams wishing to start a MIMVS program. These factors may create a hurdle for teams evaluating an eventual start of a MIMVS program.

In addition, the ISMICS consensus paper of 2011 [4] indicated longer operative times with higher incidences of stroke, aortic dissection, phrenic nerve palsy, and groin infection rates in order to reach a reduction in intensive care unit and hospital stay, transfusion rate, incidence of postoperative atrial fibrillation, and ventilation time. No wonder that teams evaluating a potential transition to MIMVS express reluctance to do so.

The present analysis challenges the existing literature and studies the outcome across Europe of real world practices in teams performing MIMVS on a regular basis. Because adopting any new technique requires a learning curve, we excluded these individual curves from the present analysis. In line with the recently suggested length of the learning curve between 35 and 75 cases $[22,23]$, we defined the learning curve at 50 cases. All coauthors estimated this to be a reasonable number, although some subjectiveness is certainly not excluded. We also included combined and reoperative cases.

Applying these criteria, we found very favorable outcome data. The 30 -day mortality rate was $1.4 \%$, which is at the lower end of reported rates for primary isolated mitral valve surgery $(0.2$ to $11.6 \%)$. In addition, the major stroke rate (modified Ranking Score $\geq 2$ ) was only $0.8 \%$ (compared with $0.6 \%$ to $4.4 \%$ reported so far) and no aortic dissections occurred. These findings are in sharp contrast with the ISMICS consensus findings [4].

While we can speculate why the results reported here are better than results reported so far, some factors may play a role. First, all centers participating in this analysis made at some point a definite decision to go for MIMVS. They were all fully experienced with mitral valve surgery as such and made a consistent change to MIMVS. This means that they were really dedicated to do so and performed MIMVS on a regular basis, albeit introducing some case selection in the transition period to MIMVS as their standard technique for mitral valve surgery. We believe it is crucially important to perform MIMVS regularly during the learning phase in order to reach reproducible results.

Next, the current analysis excludes the learning curve of the various centers involved. This may have influenced the outcome data in a favorable way. While it is not always clear in reported manuscripts if a total patient population (including the learning curve) is considered, one may assume so if it is not explicitly mentioned that the learning phase is excluded. To account for this potential bias, we considered "real daily life" data by also including combined (with tricuspid or atrial fibrillation 
surgery) and reoperative cases. Even doing so, the outcome data reported are excellent in comparison with the existing literature.

Another potential influencing factor may be the type of endo-balloon used. While most reports on MIMVS using the endo-aortic balloon technique used the Endoclamp, the current series used a mixture of Endoclamp and IntraClude devices. The IntraClude has some different features to enhance manipulation, but above all also has a precurved zone in order to facilitate aortic arch passage and limit balloon movement once inflated above the sinotubular junction. It is speculated that these features may enhance the clinical behavior of this type of endoballoon. However, this is unproven to date and is currently the topic of further research.

At first glance, a mitral valve repair rate of $73.8 \%$ may seem low to readers. However, one needs to consider that more than $10 \%$ of the cases were reoperations and that the patient population included both rheumatic and degenerative valve pathology. Four of the participating centers are located in southern Europe where the prevalence of rheumatic heart disease is still substantial. While occasional attempts for valve repair are made, most of these valves are replaced.

\section{Limitations}

The current manuscript aims to report the "real world experience" in MIMVS using a right minithoracotomy and endo-aortic balloon occlusion. We did not include centers which perform robotic MIMVS using the endoaortic balloon. However, since the principles of patient selection in robotic MIMVS and endo-aortic balloon occlusion are similar to the population studied, we do not anticipate any major outcome changes with the current results.

Our patient follow-up is limited to 30 days or inhospital time, whichever comes last. We recognize this may seem short but the scope of the current study was mainly to study outcomes related to the usage of the endo-aortic balloon. We therefore believe the study period should suffice to detect any adverse outcomes related to this technique.

The current manuscript reports on the usage of the endo-aortic balloon technique. Both the Endoclamp and IntraClude were used for aortic occlusion. The IntraClude has incorporated some design changes (easier manipulation, precurved for easier arch passage, etc) which may have influenced the outcomes in a positive way. The current analysis, however, was unable to account for this.

Although the aim of the study was also to compare the current outcome data with other techniques of mitral valve surgery, we compared our outcomes with the existing literature rather than to set up a comparative or controlled trial. This may influence the results somewhat as study populations may not be fully comparable. Nevertheless, the current study population is by no means a selected low-risk first time population undergoing MIMVS; combined and redo procedures were included making this a real world report on MIMVS. The fact that we compared the current outcomes with published data on primary isolated mitral valve surgery does certainly warrant against a lower risk population studied in this paper. In fact, the opposite should be true.

\section{Conclusions}

Once procedural proficiency is acquired, endoaortic balloon clamping in MIMVS is a safe and effective technique. Despite the fact that this patient cohort also includes combined and redo procedures, the observed mortality $(1.4 \%)$ and major stroke rate $(0.8 \%)$ compared very favorably to the existing literature on primary isolated mitral valve surgery irrespective of the approach. We believe these data strengthen the arguments to establish and expand a MIMVS program.

Edwards Lifesciences provided statistical support. All authors are members of the Medical advisory board on minimally invasive cardiac surgery by Edwards Lifesciences. Edwards Lifesciences provided reasonable travel, lodging, and honorarium for participation by many of the authors in a medical advisory board meeting where, as one of the agenda topics, initial concepts for the minimally invasive manuscript were validated. Authors were not compensated for any time spent writing or reviewing this manuscript and claim full responsibility for the integrity of this work.

\section{References}

1. DiBardino DJ, ElBardissi AW, McClure RS, RazoVasquez OA, Kelly NE, Cohn LH. Four decades of experience with mitral valve repair: analysis of differential indications, technical evolution, and long-term outcome. J Thorac Cardiovasc Surg 2010;139:76-84.

2. Aklog L, Adams DH, Couper GS, Gobezie R, Sears S, Cohn LH. Techniques and results of direct-access minimally invasive mitral valve surgery: a paradigm for the future. J Thorac Cardiovasc Surg 1998;116:705-15.

3. Mazine A, Pellerin M, Lebon J-S, Dionne P-O, Jeanmart H, Bouchard D. Minimally invasive mitral valve surgery: influence of aortic clamping technique on early outcomes. Ann Thorac Surg 2013;96:2116-22.

4. Falk V, Cheng DCH, Diegeler A, et al. Minimally invasive versus open mitral valve surgery: a consensus statement of the International Society of Minimally Invasive Coronary Surgery. (ISMICS) 2010. Innovations (Phila) 2011;(6):66-76.

5. Casselman FP, Van Slycke S, Dom H, Lambrechts DL, Vermeulen Y, Vanermen H. Endoscopic mitral valve repair: feasible, reproducible, and durable. J Thorac Cardiovasc Surg 2003;125:273-82.

6. Farrell B, Godwin J, Richards S, Warlow C, et al. The United Kingdom transient ischaemic attack (UK-TIA) aspirin trial: final results. J Neurol Neurosurg Psychiatry 1991;54:1044-54.

7. Core Team R. R: A language and environment for statistical computing. R Found dor Stat Comput 2013: Available at www.R-Project.org. Last accessed November 16, 2014.

8. Gammie JS, Sheng S, Griffith BP, et al. Trends in mitral valve surgery in the Unites States: results from the Society of Thoracic Surgeons adult cardiac database. Ann Thorac Surg 2009;87:1431-9.

9. Ryan WH, Brinkman WT, Dewey TM, Mack MJ, Prince SL, Herbert MA. Mitral valve surgery: comparison of outcomes in matched sternotomy and port access groups. J Heart Valve Dis 2010;19:51-9.

10. LaPietra A, Santana O, Mihos CG, et al. Incidence of cerebrovascular accidents in patients undergoing minimally invasive valve surgery. J Thorac Cardiovasc Surg 2014;148: $156-60$. 
11. Korn-Lubetski I, Oren A, Asher E, et al. Strokes after cardiac surgery: mostly right hemispheric ischemic with mild residual damage. J Neurol 2007;254:1708-13.

12. Shuhaiber JS, Anderson RJ. Meta-analysis of clinical outcomes following surgical mitral valve repair or replacement. Eur J Cardiothorac Surg 2007;31:267-75.

13. Modi P, Hassan A, Chitwood WR Jr. Minimally invasive mitral valve surgery: a systematic review and meta-analysis. Eur J Cardiothorac Surg 2008;34:943-52.

14. Goldstone AB, Woo YJ. Minimally invasive surgical treatment of valvular heart disease. Semin Thoracic Surg 2014;26: 36-43.

15. Mariscalco G, Musumeci F. The minithoracotomy approach: a safe and effective alternative for heart valve surgery. Ann Thorac Surg 2014;97:356-64.

16. Sundermann SH, Sromicki J, Rodriguez Cetina Biefer H, et al. Mitral valve surgery: right lateral minithoracotomy or sternotomy? A systematic review and meta-analysis. J Thorac Cardiovasc Surg J Thorac Cardiovasc Surg 2014;148:1989-95.

17. Aybek T, Dogan S, Risteski PS, et al. Two hundred forty minimally invasive mitral operations through right minithoracotomy. Ann Thorac Surg 2006;81:1618-24.

18. Galloway AC, Schwartz CF, Ribakove GH, et al. A decade of minimally invasive mitral repair: long-term outcomes. Ann Thorac Surg 2009;88:1180-4.

19. Perier P, Hohenberger W, Lakew F, Batz G, Diegeler A. Rate of repair in minimally invasive mitral valve surgery. Ann Cardiothorac Surg 2013;2:751-7.

20. Navia JL, Cosgrove DM III. Minimally invasive mitral valve operations. Ann Thorac Surg 1996;62:1542-4.

21. Gammie JS, Zhao Y, Peterson ED, O’Brien SM, Rankin JS, Griffith BP. J. Maxwell Chamberlain Memorial Paper for adult cardiac surgery. Less-invasive mitral valve operations: trends and outcomes from the Society of Thoracic Surgeons Adult Cardiac Surgery Database. Ann Thorac Surg 2010;90: 1401-10.

22. De Praetere H, Verbrugghe P, Rega F, Meuris B, Herijgers P. Starting minimally invasive valve surgery using endoclamp technology: safety and results of a starting surgeon. Interact Cardiovasc Thorac 2015;20:351-8.

23. Holzhey DM, Seeburger J, Misfeld M, Borger MA, Mohr FW. Learning minimally invasive mitral valve surgery: a cumulative sum sequential probability analysis of 3895 operations from a single high volume center. Circulation 2013;128: 483-91.

\section{Appendix}

All complications, including death, were monitored within 30 days after the operation

1. Postoperative blood transfusion:

Packed cells transfused after the operation were recorded

2. Reoperation for bleeding

Any surgical reexploration (minithoracotomy or full sternotomy) required for suspected bleeding during the postoperative period [7].

3. TIA [transitory ischemic attack]

As proposed by the STS [Society of Thoracic Surgeons] database, TIA is defined as a loss of neurologic function that was abrupt in onset but with complete return of function within 24 hours.

4. Stroke

As proposed by the STS database, a postoperative stroke is defined as any confirmed neurologic deficit of abrupt onset caused by a disturbance in blood supply to the brain, that did not resolve within 24 hours.

Stroke is separated into 2 groups:

Minor: Modified Rankin score $<2$ at 30 days

Major: Modified Rankin score $\geq 2$ at 30 days

5. Respiratory failure - Pneumonia

Severe respiratory failure is defined as intubation $>24$ hours or the patient requires reintubation for severe respiratory failure

6. Acute severe renal failure

An increase in creatinine to twice the preoperative value or the patient receives renal replacement therapy.

7. Myocardial infarction (MI)

Acute MI is present if $\leq 7$ days from the last documented MI or evolving, if, at the time of surgery, $\mathrm{Q}$-waves or ST changes were present along with a CK-MB $>5 \%$ of total CPK [7]. If patient received concomitant atrial fibrillation ablation, cardiac biomarkers were not used. Only clinical signs and electrocardiogram changes will be analyzed.

8. Peripheral ischemic event-Major vascular complication Major vascular complications include:

$\checkmark$ Any aortic dissection.

Aortic rupture.

Annulus rupture.

Left ventricle perforation.

New apical aneurysm/pseudoaneurysm.

Access site or access-related vascular injury (dissection, stenosis, perforation, rupture, arteriovenous fistula, pseudoaneurysm, hematoma, irreversible nerve injury, compartment syndrome, percutaneous closure device failure) leading to death, life-threatening or major bleeding, visceral ischemia or neurologic impairment.

Distal embolization (non-cerebral) from a vascular source requiring surgery or resulting in amputation or irreversible end-organ damage.

The use of unplanned endovascular or surgical intervention associated with death, major bleeding, visceral ischemia, or neurologic impairment.

Any new ipsilateral lower extremity ischemia documented by patient symptoms, physical exam, or decreased or absent blood flow on lower extremity angiogram.

Surgery for access site-related nerve injury OR permanent access site-related nerve injury [8].

Aortic dissection will be recorded separately in the database.

9. Surgical site infection

Any severe sterna infection requiring site reexploration (resternotomy if conversion occurred). 